\title{
Combination of Aerobics and Resistance Training to Obesity University Student Serum Visfatin Level Influence
}

\author{
Xuhui $\mathrm{Li}^{1}$ and Xiaomei Fan ${ }^{2, *}$ \\ ${ }^{1}$ College of Physical Education, Inner Mongolia Normal University, Hohhot 010022, China; ${ }^{2}$ Physiological Staff Room \\ of Basic Medical College, Inner Mongolia Medical University, Hohhot 010059, China
}

\begin{abstract}
Purpose: }}$ : Discuss on combination of long-term aerobics and resistance training to obesity university student serum visfatin level and blood lipid metabolism influence, and reveal potential mechanism of losing weight through exercises. Method: Divide 30 obesity university students into 3 groups, control group (group C), aerobics group (group A as well as aerobics and resistance training combinative group (group A+R), take in 16 periods' training, before and after intervention respectively test the three groups' weight $(\mathrm{W})$, body mass index (BMI), fat mass $(\mathrm{FM})$, fat percentage $(\% \mathrm{~F})$; serum TG, TCH, HDL-C, LDL-C content and visfatin level, result:16 after 16 weeks sports intervention, compare group $\mathrm{A}$ and group $\mathrm{A}+\mathrm{R}$ with group $\mathrm{C}, \mathrm{W}, \mathrm{BMI}, \mathrm{FM}, \% \mathrm{~F}$ obvious drop $(\mathrm{P}<0.05)$; serum TG, TCH, LDL-C content obvious drop $(\mathrm{P}<0.05)$, serum HDL-C obvious rises $(\mathrm{P}<0.05)$, serum visfatin content obvious drops $(\mathrm{P}<0.05)$, compare group $\mathrm{A}+\mathrm{R}$ with group $\mathrm{A}$, the $\mathrm{FM}, \% \mathrm{~F}$ and serum visfatin content obvious drop, other indicators have no significant differences. Relevant analysis finds that serum visfatin content is positive correlated to W, BMI, FM, F\%, TG, TCH and LDL-

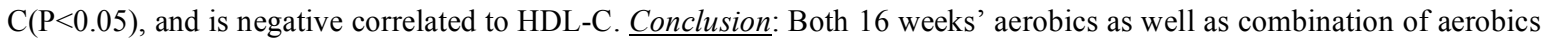
and resistance training have good improvements to obesity university students' weight and body composition, and have good adjustment on blood lipid metabolic disorder, and reduce serum visfatin level. Combination of aerobics and resistance training has more obvious impacts on $\mathrm{FM}, \% \mathrm{~F}$ and serum visfatin content, and meanwhile, serum visfatin level is positive correlated to weight, $\mathrm{BMI}, \% \mathrm{~F}, \mathrm{FM}, \mathrm{TG}, \mathrm{TCH}, \mathrm{LDL}-\mathrm{C}$, while is negative correlated to HDL-C.
\end{abstract}

Keywords: Obesity university students, resistance training, visfatin.

\section{INTRODUCTION}

Fat tissue can secrete fat cell factors of multiple bioactivity, more researches are on Leptin, Adiponectin, Ghrelin and so on, while Visfatin is a kind of fat cell factor that has found in recent years, its structure is similar to pre-B cell colony enhancement factor, which mainly has lots of expressions in visceral fat and so is called visfatin. Visfatin is closely linked to fat and can promote fat cell synthesis and aggregation, affects engine body glucolipid metabolism, so it may be one fat factor that relates to obesity and diabetes, whose relations with obesity also are always the hotspots of disputes, by far, researches on exercises to obesity university student Visfatin level have not yet been reported, the research discusses and formulates as well as analyzes inner Mongolia Normal University obesity university students' serum Visfatin level and body shape, body fat distribution, blood lipid metabolism relationships as well as aerobics and strength training intervention on them to provide experiment basis for obesity university student losing weight through exercises [1].

\section{RESEARCH OBJECTS AND METHODS}

Inner Mongolia Normal University freshman newly enrolled obesity university students volunteers are 30 people, from which men are 15 people, women are 15 people, body

\footnotetext{
*Address correspondence to this author at the Physiological Staff Room of Basic Medical College, Inner Mongolia Medical University, Hohhot 010059, China; Tel: +86-15947515306; E-mail: 10961291@qq.com
}

mass index $\geq 25 \mathrm{~kg} / \mathrm{m} 2$, randomly divide them into three groups, every group 10 people, five men and five women, the three groups are respectively control group (group C), aerobics intervention group (group A) and aerobics and resistance training combinative group (group $\mathrm{A}+\mathrm{R}$ ), take in 8 periods' exercises intervention, before intervention all take professional doctor physical examination and medical history consulting, obtain subjects personal agreement and sign written consent. For control group, except for participating school internal regulated physical education course, no extra physical exercises, while for group A and group $\mathrm{A}+\mathrm{R}$, except for participating in school internal regulated physical education course, they also should conduct 16 weeks exercises intervention according to set exercising plan, before experiment compare three groups' of students basic information, three groups of students' height, weight, body mass index, body fat percentage, body fat content, serum TG, TCH, HDL-C, LDL-C and serum Visfatin have no statistical differences [2]. During experiment process, three groups of subjects diet conducts daily prandial nutrition guiding by professional nutrition personnel, energy supply proportion is nearly as sugar $60 \% \sim 70 \%$, fat $10 \% \sim 15 \%$, protein $20 \% \sim 25 \%$, ensures balanced and reasonable nutrient energy supply, as well as diversified food source, and takes regular health education lectures per two weeks [3]. 


\subsection{Research Methods}

Exercise plan: Exercise groups' exercise plan includes aerobics and resistance training two parts. Aerobics is scheduled from 18:00 to 19:10 in the afternoon, including aerobics, jogging and sports game. Exercises intensity is controlled at $60 \%-70 \%$ of maximum heart rate. It trains 3 times per week, and $70 \mathrm{~min}$ (warm-up for $10 \mathrm{~min}$. formal training for $50 \mathrm{~min}$. relaxation training for $10 \mathrm{~min}$ ) per time. Monitoring adopts heart rate and combines with self-testing pulse, resistance training is 2 times per week, it mainly includes shoulder joint, elbow joint, hip joint, knee joint and trunk muscle groups' extension and flexion exercising, exercises intensity is nearly $65 \%-70 \%$ per time that is the maximum repeat load, conduct three groups per time. Every group includes six exercises links. Every link repeats 6-8 times, adopts minimum resistance test value (7-10RM) to predict one time maximum repeat load, calculation formula is $1 \mathrm{RM}=1.554 \times(7-10 \mathrm{RM}$ weight $)-5.185$, so as to ensure exercise plan safety and validity. Carry on medical supervision on training process. Exercise groups, after 16 weeks' exercise plan training, exercise group and control group measured relevant indicators are as following [4].

Weight, body mass index, body fat percentage and body fat content measurement:During the day before exercise intervention and the day after exercise intervention ending, it measures weight (W), body mass index (BMI), body fat percentage $(\% \mathrm{~F})$ and body fat content $(\mathrm{FM})[5]$.

Serum TG, TCH, HDL-C, LDL-C and serum Visfatin measurement:Respectively in the morning in the day before 16 weeks' exercises and in the morning in the next day after 16 weeks' exercises end, take two groups of subjects' elbow venous blood $5 \mathrm{ml}$, and serum to save in $-70^{\circ} \mathrm{C}$ with an empty belly; use fully automatic biomechanical analyser to test plasma triglyceride (TG), total cholesterol (TCH), high density lipoprotein cholesterol (HDL-C) and low density lipoprotein cholesterin (LDL-C), kit is provided by Nanjing Jiancheng biology limited company. Serum Visfatin test uses Visfatin kit to measure, kit is provided by American Rapidbio (RB) Company [6].

Statistics handling All data adopts spss13.0 software to statistic, adopts $t$ to test, $p<0.05$ represents differences have statistical significances. Serum Visfatin kit measurement changes and weight, BMI, FM, \%F, TG, TCH, LDL changes adopt correlation analysis.

\subsection{Research Results}

\subsubsection{Weight, Body Mass Index, Body Fat Percentage and Body Fat Content Changes}

By 16 weeks' exercises intervention, compare group $\mathrm{A}+\mathrm{R}$ and $\mathrm{A}$ with control group $\mathrm{C}, \mathrm{W}, \mathrm{BMI}, \mathrm{FM}, \% \mathrm{~F}$ obvious drop $(\mathrm{P}<0.05)$, compare group $\mathrm{A}+\mathrm{R}$ with group $\mathrm{A}, \mathrm{FM}$ and $\% \mathrm{~F}$ obvious drop $(\mathrm{P}<0.05)$, and $\mathrm{W}$ and $\mathrm{BMI}$ have no statistics differences, all indicators from control group before and after experiment have no statistics differences (refer to Table 1).

\subsubsection{Serum TG, TCH, HDL-C, LDL-C and Serum Vis- fatin Content Changes}

Through 16 weeks exercises, compare group $\mathrm{A}+\mathrm{R}$ and $\mathrm{A}$ with control group $\mathrm{C}$, serum TG, TCH and LDL-C level obvious drop, $(\mathrm{P}<0.05)$, HDL-C level obvious rises $(\mathrm{P}<0.05)$; group $\mathrm{A}+\mathrm{R}$ and $\mathrm{A}$ after exercising, Visfatin obviously drops $(\mathrm{P}<0.05)$ and compare group $\mathrm{A}+\mathrm{R}$ with group A, Visfatin more obvious drops $(\mathrm{P}<0.05)$, but compare group $\mathrm{A}+\mathrm{R}$ with group $\mathrm{A}$, blood lipid parameter has no statistics differences $(\mathrm{P}<0.05)$.

\subsubsection{Serum Visfatin Content and Other Indicators Corre- lations}

Before and after 16 weeks' exercise intervention, serum Visfatin levels changes respectively are positive correlated to weight, BMI, FM, \%F, TG, TCH, LDL-C changes $(\mathrm{p}<0.05)$, and are negative correlated to HDL-C changes $(p<0.05)$.

\section{ANALYSIS AND DISCUSSION}

\subsection{Before and after 16 Weeks' Exercises Weight, Body Mass Index, Body Fat Percentage and Body Fat Con- tent Changes}

Long time aerobics exercise is best plan in losing weight through exercises, and recent years' researches think that combine aerobics with medium and small intensity resistance strength training is more effective to weight losing, because aerobics can effective speed up caloric consumption, but after aerobics ending, it cannot continue to improve body basic metabolism rate for a long time, and strength training can increase gross muscle volume, increase lean body mass and then let body basic metabolism rate to be improved, which can further improve human body energy consumption under resting state [7]. The research's aerobics sports intensity is $60 \%-70 \%$ of subject maximum heart rate,

Table 1. Weight (W), body mass index (BMI), body fat percentage (\%F) and body fat content (FM) changes.

\begin{tabular}{|c|c|c|c|c|}
\hline & W (kg) & BMI(kg/m2) & FM (kg) & $\%$ F (\%) \\
\hline Group C Before & $57.15 \pm 13.24$ & $30.47 \pm 4.12$ & $31.47 \pm 2.57$ & $28.14 \pm 2.98$ \\
\hline After & $58.01 \pm 9.58$ & $28.35 \pm 3.14$ & $31.98 \pm 2.58$ & $28.99 \pm 3.54$ \\
\hline Group A Before & $57.78 \pm 11.43$ & $31.09 \pm 3.98$ & $31.87 \pm 2.89$ & $28.78 \pm 2.65$ \\
\hline After & $52.43 \pm 7.67 * \#$ & $26.65 \pm 2.76^{*} \#$ & $26.45 \pm 3.68 * \#$ & $25.01 \pm 2.67 * \#$ \\
\hline Group A+R Before & $59.0 \pm 10.13$ & $29.43 \pm 3.65$ & $32.34 \pm 2.43$ & $29.26 \pm 3.67$ \\
\hline After & $51.58 \pm 8.56^{* \#}$ & $25.21 \pm 2.43^{*} \#$ & $24.65 \pm 3.98^{*} \# \Delta$ & $23.51 \pm 2.98 * \# \Delta$ \\
\hline
\end{tabular}

Note : Before and after intervention, intra-group comparison * $\mathrm{P}<0.05$; compare group $\mathrm{A}+\mathrm{R}$ and $\mathrm{A}$ with group $\mathrm{C} \# \mathrm{P}<0.05$;Compare group $\mathrm{A}+\mathrm{R}$ with group $\mathrm{A} \Delta \mathrm{P}<0.05$. 
in the aspect of sports time, in order to stimulate fat tissue mobilization and decomposition to maximum limitation, generally it suggests sports time not to lower than 30minutes. The experiment subjects' exercises time design is 70 minutes, after 16 weeks' exercise intervention, group A and group $\mathrm{A}+\mathrm{R}$ weight, body mass index, body fat percentage and body fat content significantly drop, which is supposed that obesity university students body fat tissue speeds up mobilization and decomposition and oxidation energy supply, as well as fat constructive metabolism drop under the stimulation of exercises during 16 weeks' combination of aerobics and resistance strength training process, the research finds that compare group $\mathrm{A}+\mathrm{R}$ with group $\mathrm{A}$, group $\mathrm{A}+\mathrm{R} \mathrm{FM}, \% \mathrm{~F}$ are obvious lower than group $\mathrm{A}$, but $\mathrm{W}$ and BMI have no statistical differences, which may be strength training lets obesity students' lean body mass to obvious increase, decrease fat and meanwhile increase muscle, on a whole, compare subject weight and BMI with purely aerobics group A, they have no statistical differences, but fat mass FM and body fat percentage \%F obvious increase, for combining medium intensity aerobics with strength training, it can more effective enhance weight losing effects potential mechanism and let aerobics to be able to effective speed up caloric consumption, but after aerobics ending, it cannot continue to increase human body basic metabolism rate for a long time, while combine with strength training can increase gross muscle volume, increase lean body mass and then let human body basic metabolism rate to be promoted, which can further improve human body energy consumption under resting state [8].

\subsection{Before and After 16 Weeks' Exercise Serum TG, TCH, HDL-C, LDL-C Content Changes}

Regarding reports of long-term aerobics exercises influences on TG, TCH, HDL-C, LDL-C is inconsistent to previous reports, Jia Lei researched and found swimming could reduce nutritional obesity mice body mass, body fat, reduce serum's TCH, and LDL-C, rise HDL-C level, Huang Ya-Ru researches found 4 weeks' exercises could obvious reduce obesity youth weight, body fat, fat free mass, TCH, LDL, and improve HDL; Ji Zhi-Yong and other researched and found that after 7 weeks' aerobics, hyperlipemia rat serum TCH, TG, LDL-C content obvious declined, HDL-C obvious rose. For above result, the writer thinks that exercises influences on blood lipid metabolism may go through following paths, firstly aerobics reduced plasma's TG, TCH levels, promoted TG transportation and degradation, secondly aerobics could let cholesterol reverse transportation ability to increase, which caused blood LDL-C reducing and HDL-C rising, by above path, it drove body blood lipid metabolism improvements [9].

But regarding aerobics exercises to blood fat influences, there are also some different reports, Li Gai-Cheng and others researched and found that after 12 weeks' aerobic bodybuilding exercises, simple obesity women plasma triglyceride obviously declined, while TG, TCH, LDL-C and HDL-C had no obvious changes. Guo Yin and others observed 4 weeks' aerobics influences on obesity children blood fat, and found LDL and TC declined, but HDL-C had no significant improvements (Table 2).
After 16 weeks' exercises intervention, the research compares group $\mathrm{A}+\mathrm{R}$ and $\mathrm{A}$ with control group $\mathrm{C}$, serum TG, TCH and LDL-C levels obvious decline $(\mathrm{p}<0 \cdot 05)$, HDL$\mathrm{C}$ level obvious rises $(\mathrm{P}<0.05)$. The research thinks that before and after experiment blood lipid metabolism indicators changes have following possibilities, firstly, longterm aerobics exercises can propel to TG transferring and degradation functions, reduce serum TG, TCH contents, secondly, long-term aerobics exercise can increase TCH reverse transferring ability, which can reduce serum's LDL$\mathrm{C}$, and meanwhile rise HDL-C level. Above blood lipid metabolism changes and meanwhile it occurs to group $\mathrm{A}+\mathrm{R}$ and $\mathrm{A}$, it indicates that no matter 16 weeks' pure aerobics or aerobics and resistance training combination, both can let obesity university students' blood lipid metabolic disorder to be well improved. The experiment also finds that compare group $\mathrm{A}+\mathrm{R}$ with group $\mathrm{A}$, blood fat indicator has no statistical differences, which may be that blood fat is sensitive to aerobics endurance training, while is not sensitive to strength training, and also may be that intervention cycling 16 weeks are too short to indicate strength training effects, which needs to be further proved in future research.

\subsection{Before and After 16 weeks' Exercise Serum Visfatin Levels Changes and Other Indicators Correlations}

Visfatin is a kind of fat cell factor that mainly secreted by people and mice visceral fat tissue in latest discovery, its structure is similar to pre-B cell colony enhancement factor that mainly greatly expressed in visceral fat, so it is called Visfatin. Visfatin is closely linked to obesity and can propel to fat cell composition and aggregation, affect body glucolipid metabolism, so it may be one fat factor that is related to obesity and diabetes. Visfatin mainly is generated by visceral fat, besides, in marrow stroma cell, activated lymphocyte, liver and muscle tissues, they are also expressions to different extents, which by far has been found in human race, mice, rat and pig and other species, and suffered multiple factors adjustment. Visfatin can also promote fat cell differentiation, maturity and accumulation, and meanwhile rise as fat content increases, Fukuhara and others find that plasma Visfatin level is positive correlated to visceral fat content, but has no significant correlations with subcutaneous fat. Shang Jing and other researches indicate that exercise can improve high fat feeding caused fat metabolic disorder and obesity, and meanwhile accompanying by visceral fat tissue visfatin mRNA expression declining. Choi and others researched and found, original obesity person that plasma, visfatin concentration was higher than control group, after 12 weeks' aerobics, visfatin level obvious declined and accordingly weight obvious declined [10].

The experiment found that no matter pure aerobics group or aerobics and resistance training combinative group's obesity university students, after 16 weeks' exercise intervention, serum visfatin level obvious declines $(\mathrm{P}<0.05)$, and control group has no differences before and after experiment, and compare aerobics and resistance training combinative group with pure aerobics group, serum visfatin level obvious declines and has no statistical significance $(\mathrm{P}<0.05)$, and serum visfatin level respectively is positive 
Table 2. Before and after intervention serum TG, TCH, HDL-C, LDL-C and Visfatin levels changes.

\begin{tabular}{|c|c|c|c|c|c|}
\hline & $\begin{array}{c}\text { TG } \\
(\mathrm{mmol} / \mathrm{L})\end{array}$ & $\begin{array}{c}\text { TCH } \\
(\mathrm{mmol} / \mathrm{L})\end{array}$ & HDL-C(mmol/L) & LDL-C(mmol/L) & Visfatin(ng/ml) \\
\hline Group C Before & $3.31 \pm 0.61$ & $5.27 \pm 1.24$ & $1.11 \pm 0.56$ & $1.31 \pm 0.47$ & $86.21 \pm 10.25$ \\
\hline After & $3.28 \pm 0.64$ & $5.30 \pm 1.52$ & $1.17 \pm 0.61$ & $1.39 \pm 0.55$ & $86.32 \pm 11.03$ \\
\hline Group A Before & $3.06 \pm 0.46$ & $5.08 \pm 1.32$ & $1.20 \pm 0.56$ & $1.31 \pm 0.47$ & $85.21 \pm 10.25$ \\
\hline Group $A+R$ Before & $3.23 \pm 0.87$ & $5.09 \pm 1.43$ & $1.18 \pm 0.70$ & $1.65 \pm 0.54$ & $85.89 \pm 12.76$ \\
\hline After & $2.43 \pm 1.13^{*} \#$ & $3.68 \pm 1.54 * \#$ & $1.42 \pm 0.57 * \#$ & $1.12 \pm 0.60 * \#$ & $54.67 \pm 9.67 * \# \Delta$ \\
\hline
\end{tabular}

Note: Before and after intervention, intra-group comparison * $\mathrm{P}<0.05$; compare group $\mathrm{A}+\mathrm{R}$ and A with group $\mathrm{C} \# \mathrm{P}<0.05$; Compare group $\mathrm{A}+\mathrm{R}$ with group $\mathrm{A} \Delta \mathrm{P}<0.05$.

Table 3. Before and after exercising serum visfatin changes correlations with weight, BMI, FM, \%F, TG, TCH, LDL changes.

\begin{tabular}{|c|c|c|c|c|c|c|c|c|}
\hline Indicator & Weight & BMI & FM & \%F & TG & TCH & LDL-C & HDL-C \\
\hline \hline $\mathrm{r}$ & 0.549 & 0.521 & 0.705 & 0.688 & 0.501 & 0.489 & 0.565 & -0.598 \\
\hline $\mathrm{p}$ & 0.005 & 0.004 & 0.003 & 0.003 & 0.011 & 0.037 & 0.031 & 0.032 \\
\hline
\end{tabular}

$\mathrm{p}<0.05$ represents differences have significances.

correlated to BMI, WHR, F\%, FINS, HOMA-IR, TC, TG, LDL-C $(\mathrm{p}<0.05)$ (Table 3$)$ and it is negative correlated to HDL-C $(\mathrm{p}<0.05)$, serum visfatin reduction may have connections with the reduction of fat secretion that is caused by aerobics reduction and body fat accumulation, visfatin secretion reduces, lipid metabolism disorder obtains certain improvements. And by far it is supposed that obesity, diabetes and others, all belong to chronic low grade mucosal inflammation diseases, so we can speculate that exercises functions on visfatin may be realized through visceral fat tissue secreted some inflammatory factors, it may be one of mechanisms that exercises improve obesity, but it needs to further verify. Therefore, it can also speculate that serum visfatin level can react obesity extent and intervention effects of losing weight through exercises, but due to visfatin content and sports relevant researches are little, relevant specific deepen mechanism is still to be further explored, which is also the shortage in the research.

\section{CONCLUSION}

Both 16 weeks' pure aerobics and the combination of aerobics and resistance training have detailed effects on obesity university students' weight losing and body composition improvements, and meanwhile it also plays certain adjustment roles in obesity university students' blood lipid metabolic disorder, both can reduce serum Visfatin levels.

Compare aerobics and resistance training combination with pure aerobics, improvement functions on obesity university students' FM, $\% \mathrm{~F}$ are more obvious, let serum Visfatin level declination to be more obvious, but improvement functions on weight, BMI, and TG, TCH, LDL-C and that of pure aerobics have no statistical differences.
Serum Visfatin content changes are positive correlated to obesity people W, BMI, FM, F\%, TG, TCH and LDL$\mathrm{C}(\mathrm{P}<0.05)$, and are negative correlated to HDL-C.

\section{CONFLICT OF INTEREST}

The authors confirm that this article content has no conflict of interest.

\section{ACKNOWLEDGEMENTS}

Inner Mongolia Autonomous Region Science and Technology Agency soft science research plan project (project number 2009GXS5D189): Experiment research and popularization and application of obesity university student weight losing through exercises.

\section{REFERENCES}

[1] K.M. Choi, J.H. Kim, and G.J. Cho, "Effect of exercise training on plasma visfatin and eotaxin levels," European Journal of Endocrinology, vol. 157, no. 4, pp. 437-442, 2007.

[2] A. Fukuhara, M. Matsuda, and M. Nishizaw, "Visfatin: a protein secreted by visceral fat that mimics the effects of insulin," Science, vol. 307, pp. 426-430, 2005

[3] Y. Huang, H. Ji, and X-C. Ge, "4 weeks exercises' cooperate with diet control to obesity youth body fat composition, blood fat influences and relevant adjusting mechanism," Chinese Sports Science and Technology, vol. 9, no. 1, pp. 46-48, 2013.

[4] L. Jia, X-J. Nie, and W. Xiao, "Huangshen polysaccharide and swimming to nutrition obesity mice blood fat and leptin level intervention experiment research," Journal of Xi'an Physical Education University, vol. 28, no. 6, pp. $710-714,2011$.

[5] Z-Y. Ji, X-P. Du, and Q. Fu, "7 weeks' aerobics to high fat diet rat body fat and blood fat influence," Journal of Shenyang Physical Culture Institute, vol. 29, no. 4, pp. 37-39, 2010.

[6] J. Li, "Aerobics and resistance training combination promotion to men obesity youth heart and blood vessel functions and potential 
mechanism capacity," Sports Science, vol. 33, no. 8, pp. 37-42, 2013.

[7] G. Liu, "Aerobics to middle-aged pure obesity women serum leptin and blood fat composition influences," Sports, vol. 54, no. 11, pp. 147-148, 2012.

[8] J.K. Sethi, and A.Vidal-Puig, "Msfatin: the missing link between intra-abdominal obesity and diabetes," Trends in Molecular Medicine, vol. 11, pp. 344-347, 2005.
[9] J. Shang, L-N. Chen, and H. Sun, "Exercise intervention to high fat feeding mice fat and rib tissue Visfatin expression influence," Modern Medical Magazine, vol. 18, no. 5, pp. 551-554, 2008.

[10] Q. Yao, "Additional physical exercises functions on Nanjing obesity university students," Sports Science and Technology, vol. 31, no. 4, pp. 102-104, 2010.

Received: May 26, 2015

Revised: July 14, 2015

Accepted: August 10, 2015

(C) Li and Fan; Licensee Bentham Open.

This is an open access article licensed under the terms of the (https://creativecommons.org/licenses/by/4.0/legalcode), which permits unrestricted, noncommercial use, distribution and reproduction in any medium, provided the work is properly cited. 